\title{
A Comparative Analysis of Ideal Body Weight Methods for Pediatric Mechanical Ventilation
}

\author{
Jeffrey R Bilharz RRT RRT-NPS, Craig R Wheeler RRT RRT-NPS, \\ Brian K Walsh PhD RRT RRT-NPS RRT-ACCS RPFT AE-C FAARC, and \\ Craig D Smallwood PhD RRT
}

\begin{abstract}
BACKGROUND: A universal method for determining ideal body weight (IBW) for the application of appropriate tidal volumes in children on mechanical ventilation is elusive. We sought to compare 3 commonly used IBW methods for subjects between ages 2 and 20 y. METHODS: Demographic data were recorded, and the IBW was calculated based on the McLaren-Read, Moore, and body mass index methods by using growth chart data from the Centers for Disease Control and Prevention. The percentage error between each IBW method and the actual body weight were calculated and reported as median (interquartile range). We decided a priori that a $\geq 10 \%$ difference between the actual body weight and IBW would be clinically important. The Wilcoxon signed-rank test was used to compare the actual body weight with the IBW. Bland-Altman analysis was used to assess the individual agreement of each IBW method with the actual body weight. The KruskalWallis test was used to detect differences among the IBW methods. RESULTS: A total of 58 subjects $(36 \%$ female) were analyzed. The median (interquartile range) percent weight error between the actual body weight and calculated the IBW was $14.8 \%(1.9-22.1 \%, P=.038), 13.8 \%(4.6-$ $23.4 \%, P=.008)$, and $12.0 \%(3.9-20.5 \%, P=.037)$; the mean biases were $2.7(95 \% \mathrm{CI}-13.4$ to $18.9) \mathrm{kg}, 3.9(95 \% \mathrm{CI}-15.1$ to 22.9$) \mathrm{kg}, 3.2(95 \% \mathrm{CI}-16.7$ to 23.1$) \mathrm{kg}$; and the numbers of subjects who would have a clinically important error were $29(55.7 \%), 29(56.9 \%)$, and $30(51.7 \%)$ for the McLaren-Read, Moore, and body mass index methods, respectively. CONCLUSIONS: The majority of the subjects demonstrated a clinically important error between the actual body weight and the IBW. The percent error increased in subjects $>25 \mathrm{~kg}$ actual body weight. These data underline the importance of obtaining height measurements and calculated IBW in pediatric patients who are mechanically ventilated. Key words: ideal body weight; McLaren-Read; Moore; body mass index; pediatric; mechanical ventilation; intensive care unit; lung-protective; growth charts; comparative analysis. [Respir Care 2018;63(9):1079-1084. @ 2018 Daedalus Enterprises]
\end{abstract}

\section{Introduction}

Lung-protective ventilation strategies have been shown to reduce mortality during adult ARDS. ${ }^{1,2}$ Key compo-

\footnotetext{
Mr Bilharz and Mr Wheeler are affiliated with the Department of Respiratory Care, Boston Children's Hospital, Boston, Massachusetts. Dr. Walsh is affiliated with Liberty University, Lynchburg, Virginia. Dr. Smallwood is affiliated with the Division of Critical Care Medicine, Department of Anesthesiology, Critical Care and Pain Medicine, Boston Children's Hospital, Boston, Massachusetts, and with the Harvard Medical School, Boston, Massachusetts.
}

The authors have disclosed no conflicts of interest. nents of lung-protective ventilation include the application of low tidal volumes $\left(\mathrm{V}_{\mathrm{T}}\right)$, elevated PEEP, and permissive hypercapnia. ${ }^{3}$ It is broadly understood that several factors

\footnotetext{
Mr Bilharz presented a version of this paper as an Editors' Choice abstract at the Open Forum of the American Association of Respiratory Care Congress, held October 4-7, 2017, in Indianapolis, Indiana.

Correspondence: Craig D Smallwood PhD RRT, Division of Critical Care Medicine, Department of Anesthesiology, Critical Care and Pain Medicine, MSICU Office, Bader 634, Boston Children's Hospital, 300 Longwood Avenue, Boston, MA 02115. E-mail: craig.smallwood@childrens.harvard.edu.
}

DOI: $10.4187 /$ respcare.06021 
affect normal $\mathrm{V}_{\mathrm{T}}$, including age, sex, height, and thorax dimensions. The physiologic rationale for titrating $\mathrm{V}_{\mathrm{T}}$ to the ideal body weight (IBW) is that normal lung volumes are a function of age, sex, and thorax anatomy. Lungprotective ventilation may also be beneficial pediatric and adult in patients who do not have ARDS but receive mechanical ventilation in the ICU or operating room. ${ }^{4,5}$

\section{See the Related Editorial on Page 1189}

Importantly, the calculation of the IBW and its use during mechanical ventilation is recommended in the pediatric ICU. ${ }^{6}$ Titration of $\mathrm{V}_{\mathrm{T}}$ relative to the IBW in the pediatric ICU is especially important because the size, height, and body composition of the population vary tremendously. However, calculation of the IBW for the titration of mechanical ventilation settings is not uniformly used.?

A universal method for determining the IBW in children on mechanical ventilation is elusive. Few studies have assessed the performance and need for IBW methods in a pediatric population. ${ }^{7}$ Therefore, we sought to compare the actual body weight with 3 commonly used methods for determining IBW in pediatric subjects: the McLaren-Read (a growth chart method), Moore (a growth chart method), and body mass index (BMI) (indexed equation based on height, sex, and age) methods. ${ }^{8}$ Further, we aimed to assess the proportion of pediatric subjects who had a clinically important difference between the IBW and actual body weight.

\section{Methods}

\section{Subjects}

We conducted a retrospective analysis on a convenience sample of subjects between 2 and 20 years of age who were admitted to a single academic children's hospital combination medical-surgical pediatric ICU and who received invasive mechanical ventilation between July 2, 2014, and June 16, 2016. Patients with scoliosis and congenital heart disease were excluded from this study. Demographic data included age, height, weight, sex, mechanical ventilation duration, ICU length of stay, hospital length of stay, and primary diagnosis (surgical, respiratory, neurologic, sepsis, gastrointestinal, immunocompromised, or other). The study was approved by the institutional review board, and the need for informed consent was waived.

\section{IBW Calculation}

Three commonly used pediatric IBW methods were used for the subjects between the ages of 2 and 20 years. The McLaren-Read method is a growth chart method in which

\section{QUICK LOOK}

\section{Current knowledge}

Using ideal body weight (IBW) to calculate tidal volumes is a common practice for adults on mechanical ventilation. However, in the realm of pediatrics, there is no clear methodology for either determining or consistently implementing an IBW calculation for appropriate tidal volumes during mechanical ventilation.

\section{What this paper contributes to our knowledge}

This study compared 3 common pediatric IBW methods to the actual body weight of pediatric subjects between the ages of 2 and $20 \mathrm{y}$. Although all 3 IBW methods yielded similar results, they also revealed a clinically important percent error from the actual body weight in more than half of the subjects analyzed. This difference was observed to increase in both frequency and scale for children who weighed $>25 \mathrm{~kg}$.

the IBW is determined by using the 50th percentile for weight based on height. ${ }^{9}$ The Moore method is a growth chart method in which the IBW is determined by first locating the percentile of the subject's height and then locating the respective percentile on the weight chart. ${ }^{10}$ The BMI method is an indexed equation based on height. ${ }^{11}$ The BMI IBW was calculated by using a third-degree polynomial equation derived from plotting the 50th percentile BMI versus age (months) data according to the Centers for Disease Control and Prevention 2-20 y of age chart ${ }^{12}$ and multiplied by the subject's height $\left(\mathrm{m}^{2}\right)$. Simplified equations were created to automate calculations with age (months) and height (m).

Equation 1: simplified IBW from BMI in male subjects

$$
\begin{aligned}
& \text { BMI }(\text { male })=18.41-0.096(\text { age }) \\
& +0.00087\left(\text { age }^{2}\right)-0.000001628\left(\text { age }^{3}\right) \\
& \text { IBW }(\text { male })=[18.41-0.096(\text { age }) \\
& \left.+0.00087\left(\text { age }^{2}\right)\right] \times\left(\text { height }^{2}\right)
\end{aligned}
$$

Equation 2: simplified IBW from BMI in female subjects

$$
\begin{aligned}
\text { BMI }(\text { female })= & 18.25-0.1058(\text { age }) \\
& +0.001062\left(\mathrm{age}^{2}\right)-0.000002345\left(\mathrm{age}^{3}\right) \\
\operatorname{IBW}(\text { female })=[18.25- & 0.106(\text { age }) \\
& \left.+0.001062\left(\mathrm{age}^{2}\right)\right] \times\left(\text { height }^{2}\right)
\end{aligned}
$$




\section{Statistical Analyses}

The mean differences between each IBW method and the actual body weight for all the subjects were then calculated and their respective percent error from the actual body weight was reported as median (interquartile range). Bland-Altman analysis was used to assess the individual agreement of each IBW method and the actual body weight. ${ }^{13}$ Because the data were not normally distributed, the Wilcoxon signed-rank test was used to compare the IBW with the actual body weight. ${ }^{14}$ The Kruskal-Wallis test was used to detect significant differences among the methods of IBW calculations by using the actual body weight as a reference. We decided a priori, that a $\geq 10 \%$ difference between the actual body weight and the IBW would be clinically important. ${ }^{7}$ Data were compiled by using MATLAB (V9.1.0.441655, Mathworks, Natick, Massachusetts), and statistical analyses were completed in Excel (V12.0 071130, Microsoft, Redmond, Washington). Prism 6.0 (GraphPad Software, La Jolla, California) was used to graph and derive the equations for the BMI IBW method and to perform the Kruskal-Wallis test.

\section{Results}

A total of 58 subjects ( $36 \%$ female) were analyzed. Demographic data included age, height, weight, sex, mechanical ventilation duration, ICU length of stay, hospital length of stay, primary diagnosis (surgical, respiratory, neurologic, sepsis, gastrointestinal, immunocompromised, or other) and reported as median (interquartile range) as depicted in Table 1. The median (interquartile) weight differences $(\%)$ between the actual body weight and calculated IBW was $14.8 \%(1.9-22.1 \%, P=.038), 13.8 \%$ $(4.6-23.4 \%, P=.008)$, and $12.0 \%(3.9-20.5 \%, P=.037)$ for McLaren-Read, Moore, and BMI methods, respectively (Table 1). The numbers of subjects who would have a clinically important error were 29 (55.7\%), 29 (56.9\%), and 30 (51.7\%) for McLaren-Read, Moore, and BMI methods, respectively (Table 1). The Kruskal-Wallis test did not detect a significant difference between the methods of IBW calculation for male or female subjects $(P=.99$ and $P=.91$, respectively). The mean biases were 2.7 (95\% CI -13.4 to 18.9) kg for McLaren-Read (Fig. 1), 3.9 (95\% CI -15.1 to 22.9) $\mathrm{kg}$ for Moore (Fig. 2), and 3.2 (95\% CI -16.7 to 23.1) $\mathrm{kg}$ for BMI (Fig. 3). The percent error increased in subjects $>25 \mathrm{~kg}$ actual body weight.

\section{Discussion}

We compared 3 common IBW methods used in the pediatric population. If the actual body weight were used instead of IBW, then the majority of the patients would have a clinically important difference in applied $\mathrm{V}_{\mathrm{T}}$. The
Table 1. Demographic, Diagnosis, and Ideal Body Weight Method Data

\begin{tabular}{lc}
\hline \multicolumn{1}{c}{ Parameter } & Results \\
\hline Age, median (IQR) mo & $106.9(52.1-164.8)$ \\
Weight, median (IQR) kg & $30.8(16.8-41.0)$ \\
Height, median (IQR) cm & $128(100-155)$ \\
Duration of ventilation, median (IQR) d & $3.5(1.4-9.7)$ \\
ICU LOS, median (IQR) d & $11.9(7.9-26.6)$ \\
Hospital LOS, median (IQR) d & $12.9(6.6-71.6)$ \\
Subjects, $N$ (\% female) & $58(36)$ \\
Primary diagnosis, $n(\%)$ & \\
Surgical & $5(8.6)$ \\
Respiratory & $11(19.0)$ \\
Neurologic & $16(27.6)$ \\
Sepsis & $5(8.6)$ \\
Gastrointestinal & $6(10.3)$ \\
Immunocompromised & $5(8.6)$ \\
Other & $10(17.2)$ \\
Ideal body weight method & \\
McLaren method & \\
$\quad$ Weight difference, median (IQR) $\%$ & $14.8(1.9-22.1)$ \\
$\quad$ Difference $\geq 10 \%, n(\%)$ & $29(55.7)$ \\
Moore method & \\
$\quad$ Weight difference, median (IQR) $\%$ & $13.8(4.6-23.4)$ \\
$\quad$ Difference $\geq 10 \%, n(\%)$ & $29(56.9)$ \\
BMI method & \\
$\quad$ Weight difference, median (IQR) $\%$ & \\
$\quad$ Difference $\geq 10 \%, n(\%)$ & \\
\hline IQR $=$ interquartile range & \\
LOS $=$ length of stay & \\
BMI $=$ body mass index & \\
Difference $\geq 10 \%$ was defined as difference between actual body weight and IBW. & \\
Other includes, other infection, orthopedic procedure and otorhinolaryngology. & \\
\hline & \\
&
\end{tabular}

projected error between the actual body weight and the IBW methods would similarly affect measured $\mathrm{V}_{\mathrm{T}} / \mathrm{kg}$ in this population. Importantly, subjects with an actual body weight of $>25 \mathrm{~kg}$ were at an increased risk for a large difference between the actual body weight and the IBW.

Martin and Richards ${ }^{7}$ defined similar percent error targets when comparing IBW models (Devine formula ${ }^{15}$ ) used in the 2000 ARDS Network study and the IBW relationship taken from the 1998 trial by Stewart et al ${ }^{16}$ against their synthesis of a unisex population median reference curve derived from 4 major data sources designed to produce the most-appropriate lung-protective $\mathrm{V}_{\mathrm{T}}$. The investigators found significant weight disparities, including up to a $30 \%$ overestimation, among the referenced IBW models. Although their study did not apply these models to the actual body weight within a studied cohort, it set forth more applicable percent weight error targets of no more than $5 \%$ above and no more than $10 \%$ below the given IBW models.

The accountancy of this possible over- and underinflation of the lungs was not as well represented in our study, 


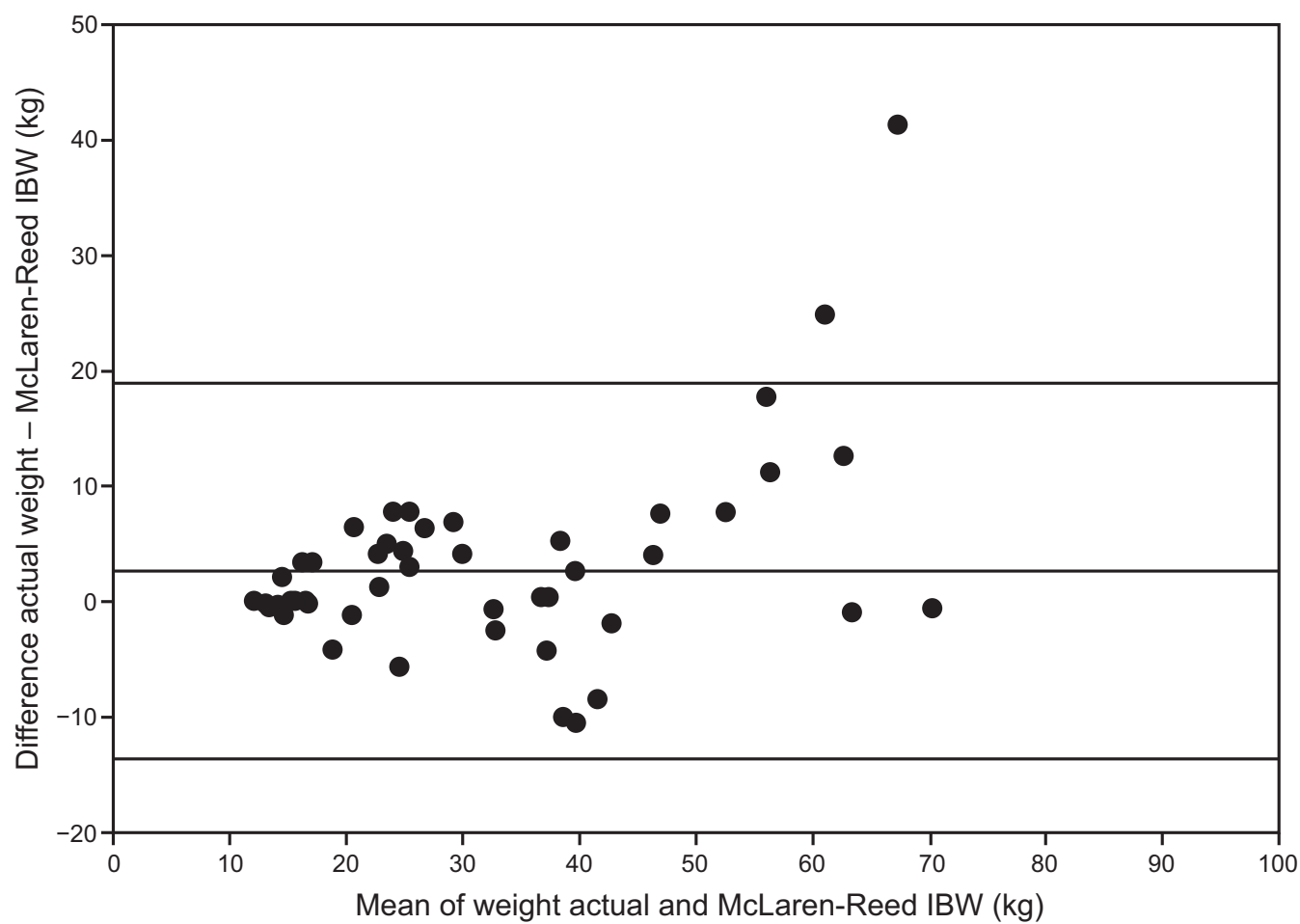

Fig. 1. Bland-Altman plot for the McLaren-Read IBW method in male and female subjects ages 2-20 y. The center line denotes bias; outside lines show upper and lower limits. IBW = ideal body weight.

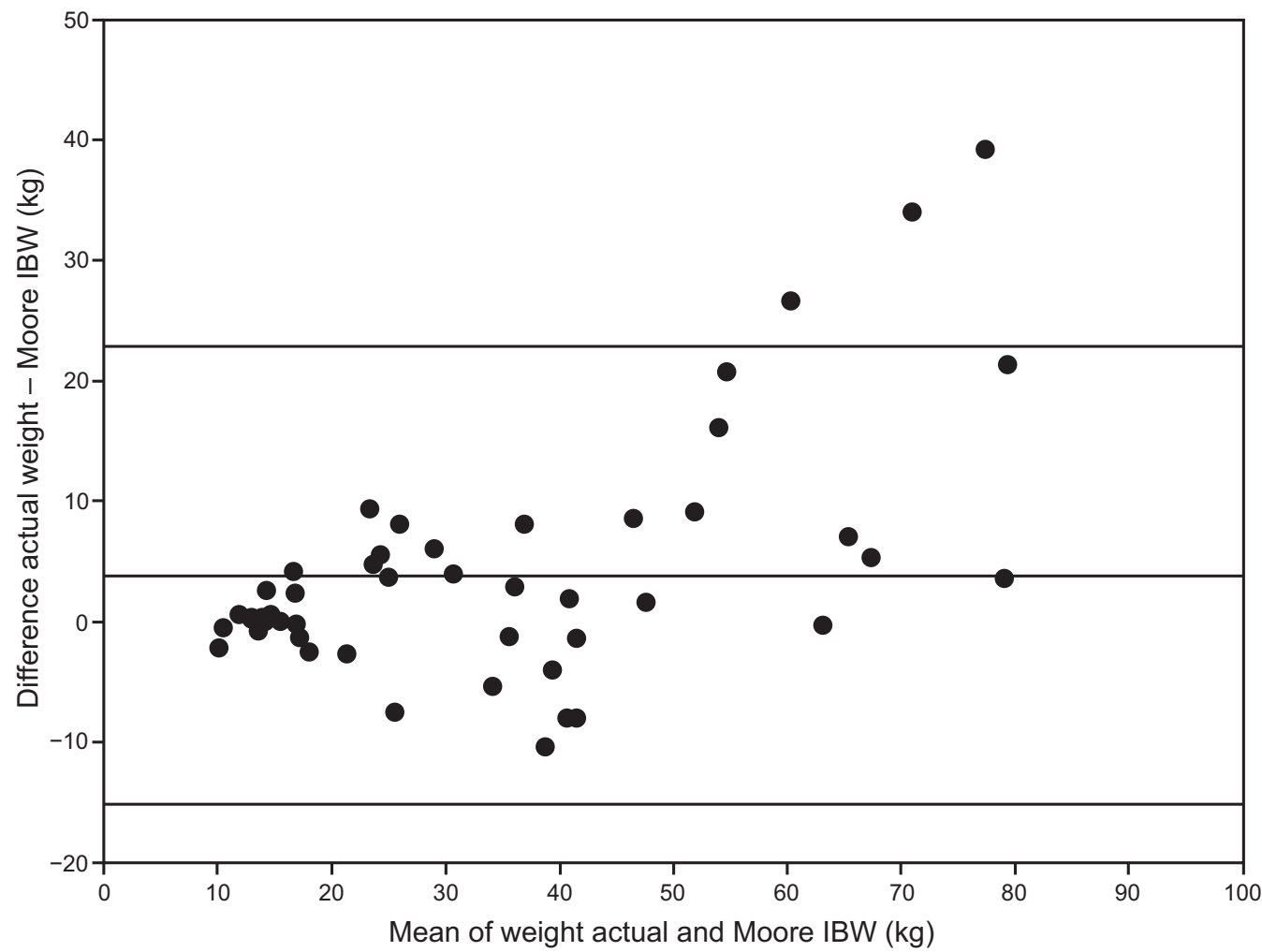

Fig. 2. Bland-Altman plot for the Moore IBW method in male and female subjects ages 2-20 y. The center line denotes bias; outside lines show upper and lower limits. IBW = ideal body weight. 


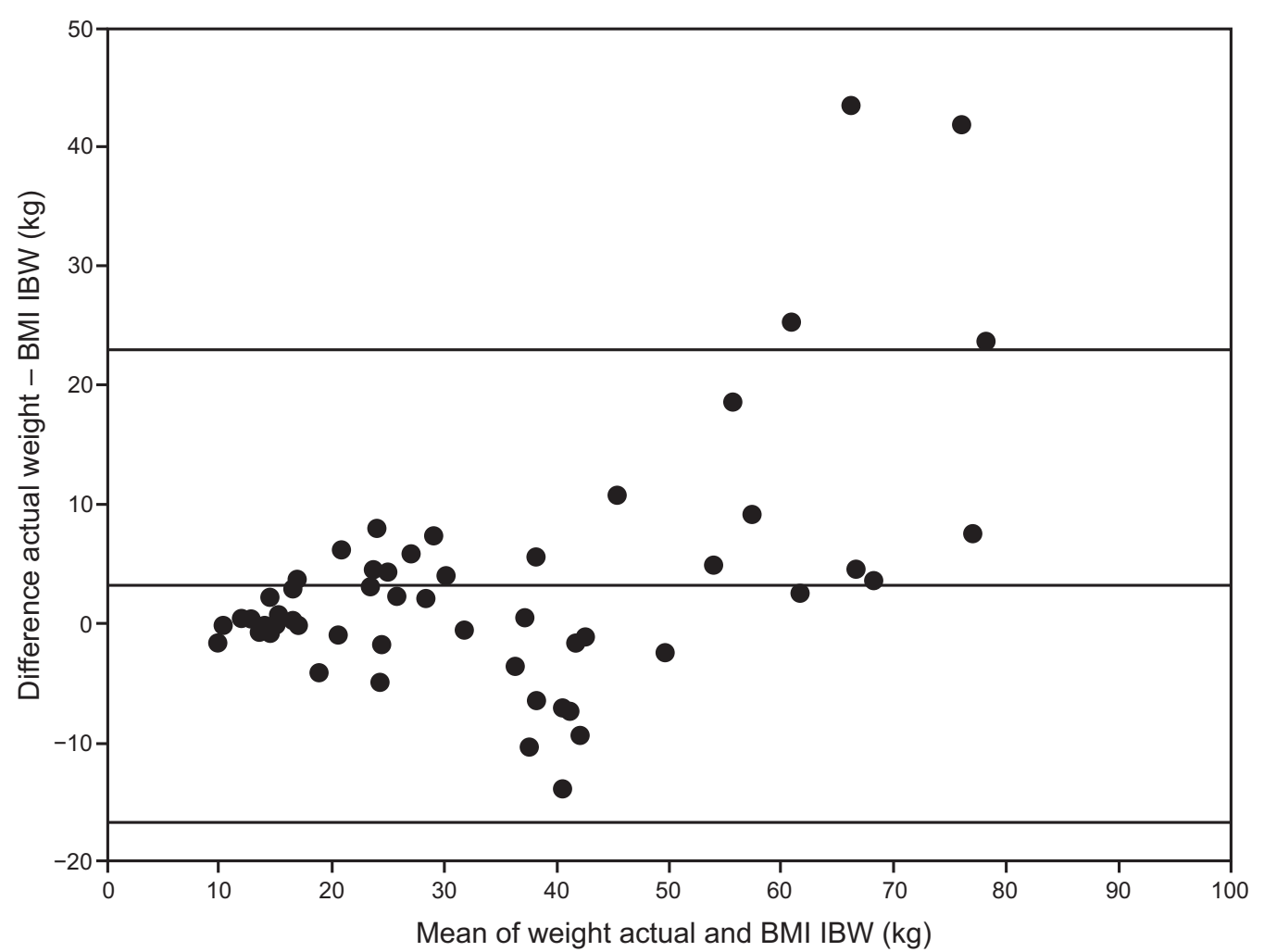

Fig. 3. Bland-Altman plot for the BMI IBW method in male and female subjects ages 2-20 y. The center line denotes bias; outside lines show upper and lower limits. BMI = body mass index; IBW = ideal body weight.

in which a clinically important error was presented as an absolute value of the percent error. Whereas the paucity of pediatric data has limited the ability to set such a similarly detailed lung-protective error range within this small-scale investigation, the consistent weight discrepancies should emphasize the use of the IBW for mechanically ventilated children. In the past, it has been suggested that the actual body weight be used for pediatric patients who weigh less than the 50th percentile for their age whereas the IBW predicted from the height or ulna length be used for those weighing above the 50th percentile. ${ }^{17}$ However, our data underscored the importance of the applied IBW for mechanical ventilation for all patients. The risk of volutrauma could potentially be mitigated across all populations by following a standardized approach to $\mathrm{V}_{\mathrm{T}}$ calculation. ${ }^{18}$

Although the use of the IBW in pediatric mechanical ventilation can be beneficial, there is some ambiguity about which method is preferred. In the present investigation, we noted a similar error among 3 IBW methods when compared with the actual body weight. There are some important practical differences among the methods assessed that should be considered. The BMI IBW has a greater height range and, therefore, may be applied to a broader range of patients relative to the other methods, but variables such as marginalized height and weight percentiles may interfere with this accuracy.
There are important limitations of the present study. First, the population was heterogeneous, and it was difficult to specifically note differences based on sex or other factors. However, the mix of diseases and subject sizes was generally reflective of a typical combined medical surgical pediatric ICU population. Second, the study was retrospective in nature and we, therefore, were not able to directly measure $\mathrm{V}_{\mathrm{T}} / \mathrm{kg}$ provided. However, because the relationship between $\mathrm{V}_{\mathrm{T}} / \mathrm{kg}$ and IBW is linear, extrapolating the error was straightforward. Third, we were unable to assess differences based on sex because the number of subjects enrolled in the study was modest. It will be important to address this feature in future work. Fourth, due to the retrospective nature of this study, we were not able to verify the height measuring technique, which is known to be difficult to accurately obtain in bedridden subjects. Fifth, the threshold for clinical importance in the present work was defined as an error of $\geq 10 \%$. This threshold may be different, depending on individual practice, patient conditions, and severity of illness. Sixth, we did not detect differences among the methods of calculating IBW, but our sample size was modest, and, therefore, it is conceivable that differences could be detected in select cohorts or with a larger sample. Seventh, we did not examine the effects of IBW on $\mathrm{V}_{\mathrm{T}}$ in children $<2 \mathrm{y}$ old, and extrapolation of 
present findings should be done with caution. It is necessary for future work to assess this population.

\section{Conclusions}

The majority of the subjects demonstrated a clinically important error between the actual body weight and the calculated IBW regardless of what IBW method was used. However, this percent error increased in subjects $>25 \mathrm{~kg}$ actual body weight for all 3 IBW methods. These data underline the importance of obtaining height measurements and calculated IBW in pediatric patients on mechanical ventilation. We recommend future mechanical ventilation research to clearly indicate a methodology for IBW calculations and their relationship to patient outcomes.

\section{REFERENCES}

1. Acute Respiratory Distress Syndrome Network, Brower RG, Matthay MA, Morris A, Schoenfeld D, Thompson BT, Wheeler A. Ventilation with lower tidal volumes as compared with traditional tidal volumes for acute lung injury and the acute respiratory distress syndrome. N Engl J Med 2000;342(18):1301-1308.

2. Petrucci N, De Feo C. Lung protective ventilation strategy for the acute respiratory distress syndrome. Cochrane Database Syst Rev 2013(2):CD003844.

3. Santschi M, Jouvet P, Leclerc F, Gauvin F, Newth CJ, Carroll CL, et al; PALIVE Investigators, Pediatric Acute Lung Injury and Sepsis Investigators Network (PALISI), European Society of Pediatric and Neonatal Intensive Care (ESPNIC). Acute lung injury in children: therapeutic practice and feasibility of international clinical trials. Pediatr Crit Care Med 2010;11(6):681-689.

4. Serpa Neto A, Simonis FD, Schultz MJ. How to ventilate patients without acute respiratory distress syndrome? Curr Opin Crit Care 2015;21(1):65-73.

5. Sutherasan Y, D'Antini D, Pelosi P. Advances in ventilator-associated lung injury: prevention is the target. Expert Rev Respir Med 2014;8(2):233-248.
6. Rimensberger PC, Cheifetz IM; Pediatric Acute Lung Injury Consensus Conference Group. Ventilatory support in children with pediatric acute respiratory distress syndrome: proceedings from the Pediatric Acute Lung Injury Consensus Conference. Pediatr Crit Care Med 2015;16(5 Suppl 1):51-S60.

7. Martin DC, Richards GN. Predicted body weight relationships for protective ventilation-unisex proposals from pre-term through to adult. BMC Pulm Med 2017;17(1):85.

8. Phillips S, Edlbeck A, Kirby M, Goday P. Ideal body weight in children. Nutr Clin Pract 2007;22(2):240-245.

9. McLaren DS, Read WW. Assessment of nutritional status of children. Lancet 1973;2(7825):374-375.

10. Moore DD, PR; Forstner, GG; Pencharz PB. The assessment of nutritional status in children. Nutr Res 1985;5(8):797-799.

11. Khadilkar V, Khadilkar A. Growth charts: A diagnostic tool. Indian J Endocrinol Metab 2011; 15(Suppl 3):S166-S171.

12. Centers for Disease Control and Prevention. 2000 CDC Growth charts: United States. https://www.cdc.gov/growthcharts/cdc_charts.htm. Accessed April 3, 2017.

13. Giavarina D. Understanding Bland Altman analysis. Biochem Med (Zagreb) 2015;25(2):141-151.

14. Datta $S$, Satten GA. A signed-rank test for clustered data. Biometrics 2008;64(2):501-507.

15. McCarron M, Devine BJ. Clinical pharmacy: case studies case number 25. Gentamicin therapy. Ann Pharmacother 1974;8:650-655.

16. Stewart TE, Meade MO, Cook DJ, Granton JT, Hodder RV, Lapinsky SE, et al. Evaluation of a ventilation strategy to prevent barotrauma in patients at high risk for acute respiratory distress syndrome. Pressure- and Volume-Limited Ventilation Strategy Group. N Engl J Med 1998;338(6):355-361.

17. Khemani RG, Newth CJ. The design of future pediatric mechanical ventilation trials for acute lung injury. Am J Respir Crit Care Med 2010;182(12):1465-1474.

18. Newth CJL, Sward KA, Khemani RG, Page K, Meert KL, Carcillo JA, et al; Eunice Kennedy Shriver National Institute for Child Health and Human Development Collaborative Pediatric Critical Care Research Network (CPCCRN). Variability in Usual Care Mechanical Ventilation for Pediatric Acute Respiratory Distress Syndrome: Time for a Decision Support Protocol? Pediatr Crit Care Med 2017;18(11):e521-e529.

This article is approved for Continuing Respiratory Care Education credit. For information and to obtain your CRCE

(free to AARC members) visit www.rcjournal.com 